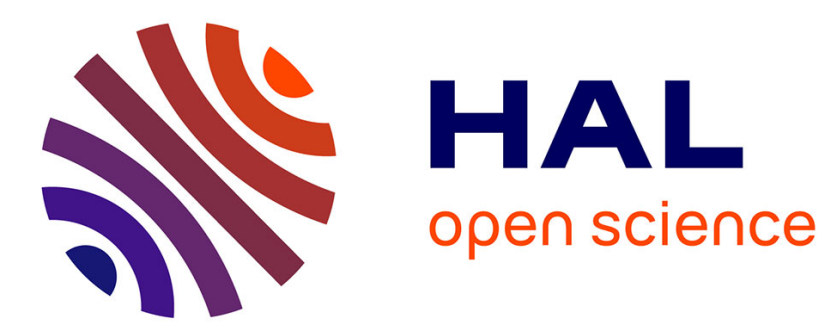

\title{
Interaction of ultrasound waves with bone remodelling: a multiscale computational study
}

Cécile Baron, Vu-Hieu Nguyen, Salah Naïli, Carine Guivier-Curien

\section{To cite this version:}

Cécile Baron, Vu-Hieu Nguyen, Salah Naïli, Carine Guivier-Curien. Interaction of ultrasound waves with bone remodelling: a multiscale computational study. Biomechanics and Modeling in Mechanobiology, 2020, 10.1007/s10237-020-01306-7 . hal-02911706

\section{HAL Id: hal-02911706 https://hal.science/hal-02911706}

Submitted on 14 Dec 2020

HAL is a multi-disciplinary open access archive for the deposit and dissemination of scientific research documents, whether they are published or not. The documents may come from teaching and research institutions in France or abroad, or from public or private research centers.
L'archive ouverte pluridisciplinaire $\mathbf{H A L}$, est destinée au dépôt et à la diffusion de documents scientifiques de niveau recherche, publiés ou non, émanant des établissements d'enseignement et de recherche français ou étrangers, des laboratoires publics ou privés. 


\title{
Interaction of ultrasound waves with bone remodelling: a multiscale computational study
}

\author{
Cécile Baron · Vu-Hieu Nguyen · Salah Naili • Carine Guivier-Curien
}

Received: date / Accepted: date

\begin{abstract}
Ultrasound stimulation is thought to influence bone remodeling process. But recently, the efficiency of ultrasound therapy for bone healing has been questioned. Despite an extensive literature describing the positive effect of ultrasound on bone regeneration cell cultures, animal models, clinical studies - there are more and more reviews denouncing the inefficiency of clinical devices based on low intensity pulsed ultrasound stimulation (LIPUS) of the bone healing. One of the reasons to cause controversy comes from the persistent misunderstanding of the underlying physical and biological mechanisms of ultrasound stimulation of bone repair. As ultrasonic waves are mechanical waves, the process to be studied is the one of the mechanotransduction. Previous studies on the bone mechanotransduction have demonstrated the key-role of the osteocytes in bone mechanosensing. Osteocytes are bone cells ubiquitous inside the bone matrix, they are immersed in the interstitial fluid (IF) inside the lacuno-canalicular network (LCN). They are considered as particularly sensitive to a particular type of mechanical stress: wall shear stress on osteocytes due to the IF flow in the LCN. Inspired from these findings and observations, the
\end{abstract}

Cécile Baron

Aix-Marseille Univ, CNRS, ISM, Marseille, France

Aix-Marseille Univ, APHM, CNRS, ISM, Sainte-Marguerite Hospital, Institute for Locomotion, Department of Orthopaedics and Traumatology, Marseille, France

E-mail: cecile.baron@univ-amu.fr

Vu-Hieu Nguyen

Univ Paris-Est, CNRS, MSME, Créteil, France

Salah Naili

Univ Paris-Est, CNRS, MSME, Créteil, France

Carine Guivier-Curien

Aix-Marseille Univ, CNRS, Centrale Marseille, IRPHE, Marseille, France present work investigates the effect of LIPUS on the cortical bone from the tissue to the osteocytes, considering that the impact of the ultrasound stimulation applied at the tissue scale is related to the mechanical stress experimented by the bone cells. To do that, simulations based on the finite element method are carried out in the commercial software Comsol Multiphysis to assess the wall shear stress levels induced by the LIPUS on the osteocytes. Two formulations of the wall shear stress were investigated based on two IF flow models inside the LCN and associated with two different values of the LCN permeability. The wall shear stress estimate is very different depending on the assumption considered. One of these two models provides wall shear stress values in accordance with previous works published on bone mechanotransduction. This study presents the preliminary results of a computational model that could provide keys to understanding the underlying mechanisms of the LIPUS.

Keywords Bone remodelling - ultrasound stimulation $\cdot$ osteocyte $\cdot$ poroelasticity $\cdot$ wall shear stress

\section{Introduction}

Bone is a living tissue that is constantly being remodelled, adapting to its mechanical environment and capable of repair. Bone is one of few tissues that can heal without forming a fibrous scar. Trauma is the most expensive medical condition after heart conditions, costing the United States 56 billion dollars every year. Of that, 21 billion is used for the treatment of fractures. For these reasons, the efficacious and expedient treatment of fractures is of paramount importance to the patient, physician, and healthcare system as a whole (Buza and Einhorn, 2016). Unfortunately, sometimes 
the healing process fails and non-unions or delayed unions occur. Delayed fracture healing and nonunion are observed in up to $5-10 \%$ of all fractures, and can present a challenging clinical scenario for the treating physician. The beneficial effect of ultrasound on bone remodelling was discovered in the 1950s (Corradi and Cozzolino, 1953; Yasuda, 1977). Since the 1980s, several authors have studied this phenomenon in different processes of adaptation of the bone to its environment: growth (Duarte, 1983), targeted remodeling (Chan et al., 2006) and healing (Schortinghuis et al., 2003).

However, within the recent years, several systematic reviews and meta-analyses (Busse et al., 2014; Schandelmaier et al., 2017; Griffin, 2016) provide moderate to high-certainty evidences against the contribution of USS in bone healing. The controversy on the subject continues and many questions remain unanswered (Aspenberg, 2017; Mortazavi et al., 2016). Actually, the real issue is that the underlying physico-biological mechanisms of the of ultrasound on bone remodeling remain unclear, as reported in several reviews (Claes and Willie, 2007; Martinez de Albornoz et al., 2011; Padilla et al., 2014). One of the objectives of this work is to gain insight into the mechanical impact of ultrasound stimulation on bone from tissue to cell and explore its capacity to trigger bone remodeling.

On one hand, clinical studies, animal models and cell cultures put on evidence an effect of LIPUS on bone repair, but the underlying physico-biological mechanisms remain unclear and feed an important literature as reported in several paper reviews (Claes and Willie, 2007; Martinez de Albornoz et al., 2011; Padilla et al., 2014). On the other hand, the influence of a mechanical loading on bone remodelling has been widely explored by Cowin et al. (1991); Weinbaum et al. (1994); Claes and Heigele (1999); Mullender et al. (2004); Klein-Nulend et al. (2013), for instance. Some of these works have revealed the osteocytes as the cornerstone of the mechanosensing and the pilots of the bone mechanotransduction. The osteocytes are ubiquituous bone cells sited in the lacuno-canalicular network (LCN), immerged in the interstitial fluid (IF) which fullfills the voids between osteocytes and the extra-cellular matrix (ECM). Authors consider that when mechanical loading is applied to bone at macroscale, it induces pressure gradients inside the bone, resulting in IF flow around the osteocytes which are in turn submitted to several mechanical loadings such as radiation forces, hydrostatic pressure or wall shear stress (WSS). The latter one has been introduced by Weinbaum et al. (1994) and experimentally demonstrated by Klein-Nulend et al. (1995) as the dominant factor, in bone mechanotransduction more than streaming for example (Klein-Nulend et al., 2005, 2013). Therefore, in this preliminary study we chose to focus on the WSS. The main assumption is that the bone remodelling is partly triggered by the level of fluid-induced wall shear stress especially on the process of the osteocytes (Bakker et al., 2001; Anderson et al., 2005).

A lot of numerical studies showed the impact of the physiological loading on the bone to interpret the mechanobiology of bone healing (Claes and Heigele, 1999; Lacroix and Prendergast, 2002; Isaksson et al., 2006; González-Torres et al., 2010; Gómez-Benito et al., 2011; Nguyen et al., 2009, 2010a, 2011). These numerical models usually considered a cyclic compressive loading applied to bone at frequency corresponding to daily activity (walk, run, jump) and related the mechanical stimulus induced at the cell scale to a biological response in terms of proliferation and differentiation of bone cells following the model of Prendergast and colleagues (Prendergast et al., 1997). Ultrasound stimulation is a type of mechanical loading and as such may trigger reactions similar to physiological loading. However the characteristics of ultrasonic loading are very different from those of physiological loading and this raises a number of questions such as: do the mechanotransduction processes identified for physiological loading also act on osteocyte at US frequencies? Are there any others phenomena which can be implied in ultrasound stimulation? To understand how ultrasound could affect bone remodelling, a computational model is developed in order to estimate the level of mechanical stimulus applied on the osteocytes by the mesoscopic ultrasound stimulation. To our knowledge, it is the first numerical study on the multiscale interaction of ultrasound with the bone remodelling process.

This paper does the link between biological observations and physical interpretation integrating both bone levels of porosity: the vascular network and the LCN network. However, the aim is not to infer the biological mechanisms acting inside the cell, but to concentrate on the characterization of the mechanical forces applied to the cell via the study of the WSS. To appropriately tackle the question, this mechanical model integrates two levels of porosity: the vascular network (Havers and Volkman canals - $100 \mu \mathrm{m}$ ) reconstructed from CT scans and the lacuno-canalicular network containing the osteocytes $(1 \mu \mathrm{m})$ taken into account through the poroelasticity theory. On the basis of previous works, this paper proposes to estimate the osteocyte wall shear stress identified as the relevant stimulus in bone mechanotransduction. To do this properly, one of the key parameters 
is the permeability of the LCN, the value of which remains controversial (Cardoso et al., 2013).

As a consequence, the goal of this paper is focused on the mechanical aspect of the bone mechanotransduction under ultrasound stimulation. The relevancy of the proposed mechanical model is tested looking at the influence of the LCN permeability value on the wall shear stress induced on the osteocytes by the ultrasound stimulation. The wall shear stress levels assessed for two permeability values are compared and discussed in relation to the results of the literature.

After this introduction, the paper is organized as follows. The materials and methods are presented in section 2. We give in subsection 2.1 the in vivo configuration, the geometry description is presented in subsection 2.2 , the governing equations are given in subsection 2.3, the weak formulation is given in subsection 2.4 and the computational model is given in subsection 2.5. We show and discuss the main results on the estimation of wall shear stress applied to the osteocyte by ultrasonic stimulation in section 3. Section 4 finishes the manuscript with the conclusion.

\section{Materials and Methods}

\subsection{In vivo configuration}

Ultrasound stimulation is mainly dedicated to fracture healing in clinical applications, however, the goal of this preliminary study is to investigate the effect of ultrasound as a trigger for bone remodelling process and not its direct influence on the healing callus. Consequently, we choose to represent a slice of intact cortical bone which can be above or below the fracture site and still in interaction with ultrasonic waves since the US transducer has a diameter of $1 \mathrm{~cm}$, greater than the fracture gap size (few $\mathrm{mm}$ ). Considering that the remodelling would be managed by the osteocytes, the bone healing should be initiated from the intact parts of bone surrounding the fracture site. Moreover, the ultrasound stimulation of bone remodelling could be used for skeletal events other than fracture (localized bone loss, bone metastases, osteolytic tumors) and could also be represented by the model developed in this study. This model is therefore a $2 \mathrm{D}$ model considering a bone cross-section perpendicular to the bone axis. Cortical bone is considered as a biphasic medium (fluid-saturated poroelastic solid) taking into account two levels of porosity. The first one is the vascular porosity level (pores $\varnothing \sim 100 \mu \mathrm{m}$ ). The vascular pores geometry is extracted from $\mu \mathrm{CT}$ images (pixel size $=22.5 \mu \mathrm{m}$ ). The vascular pores are supposed to be full of fluid. The second one is the LCN where the osteocytes are sited surrounded by the interstitial fluid. This level of porosity is constituted of an ubiquitous pore network of lacunae (pores $\varnothing \sim 15 \mu \mathrm{m}$ ) and canaliculi (pores $\varnothing \sim 0.5 \mu \mathrm{m}$ ). The size of these voids are too small to be extracted from the above-mentioned $\mu \mathrm{CT}$ images, consequently the bone matrix around the vascular pores is modeled as a poroelastic medium using Biot's theory to describe the wave propagation in an equivalent medium (Biot, $1955,1957)$. To be close to the in-vivo configuration, the model takes into account the soft tissues around the bone considered as water in first approximation.

\subsection{Geometry description}

For this problem, we consider a two dimensional (2D) plane strain model as shown in Fig. 1. In this model, the poroelastic bone domain is denoted $\Omega^{b}$. The soft tissue, the marrow and the fluid-filled lacuna are all considered as fluid and denoted by $\Omega_{0}^{f}, \Omega_{j}^{f}(j=1, . ., N-1)$ and $\Omega_{N}^{f}$, respectively. The interfaces between the bone $\Omega^{b}$ and the fluid domains $\Omega_{j}^{f}$ are denoted by $\Gamma_{j}(j=$ $0, \ldots, N)$. The external surface of the soft tissue domain $\Omega_{0}^{f}$ consists of 2 parts denoted by $\Gamma_{0}^{F}$ (free surface) and $\Gamma_{0}^{P}$ (imposed by a pressure), respectively.

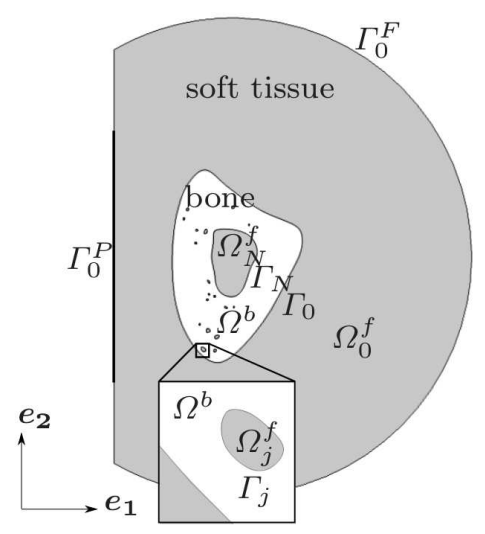

Fig. 1: Geometry description

In what follows, we denote the coordinates of a point $\boldsymbol{x}$ by $\left(x_{1}, x_{2}\right)$ and the time by $t$. A superposed dot stands for time derivative, $\nabla$ and $\nabla \cdot$ stand for the gradient and divergence operators in $2 \mathrm{D}$ space, respectively. The symbol "." denotes the scalar product and the symbol ":" between tensors of any order denotes their double contraction. 


\subsection{Governing equations}

\subsubsection{Equations in the fluid domains $\Omega_{j}^{f}$}

The fluid occupying the domain $\Omega_{j}^{f}$ is an acoustic fluid whose mass density and bulk modulus at equilibrium are denoted by $\rho_{j}^{f}$ and $K_{j}^{f}$, respectively. By using the linear acoustic theory and by neglecting the body forces (other than inertia), the wave equation for the fluid in the domain $\Omega^{j}$ may be expressed only in terms of the pressure field $p_{j}^{f}(\boldsymbol{x}, t)$ as follows:

$\frac{1}{K_{j}^{f}} \ddot{p}_{j}^{f}-\frac{1}{\rho_{j}^{f}} \nabla^{2} p_{j}^{f}=0, \quad \forall \boldsymbol{x} \in \Omega_{j}^{f} \quad(j=0, \ldots, N)$.

Note that the index $j$ is used for indicating the number of the fluid domain, then there is no summation over $j$ in the above equation as well as in the following.

The velocity vector $\boldsymbol{v}_{j}(\boldsymbol{x}, t)$ is relied to the gradient of pressure field $p_{j}^{f}(\boldsymbol{x}, t)$ following the Euler equation:

$\rho_{j}^{f} \dot{\boldsymbol{v}}_{j}+\nabla p_{j}^{f}=\mathbf{0}, \quad \forall \boldsymbol{x} \in \Omega_{j}^{f}, \quad(j=0, \ldots, N)$.

\subsubsection{Equations in the anisotropic poroelastic bone $\left(\Omega^{b}\right)$}

The cortical bone material is assumed to consist of a solid skeleton (with mass density $\rho_{s}$ ) and a connected pore network saturated by fluid (with mass density $\rho_{f}$ ). The Biot's anisotropic poroelastic model (Biot, 1957; Coussy, 2005; Thompson and Willis, 1991) could be used to describe the wave propagation problem in bone (Cowin, 2003; Nguyen and Naili, 2012).

Neglecting the body forces (other than inertia), the equations describing the wave propagation within the bone domain $\left(\Omega_{b}\right)$ read:

$\nabla \cdot \boldsymbol{\sigma}=\rho \ddot{\boldsymbol{u}}_{s}+\rho_{f} \ddot{\boldsymbol{w}}$,

$-\nabla p=\rho_{f} \ddot{\boldsymbol{u}}_{s}+\boldsymbol{\kappa}^{-1} \dot{\boldsymbol{w}}+\boldsymbol{b} \ddot{\boldsymbol{w}}$

where $\rho=\phi \rho_{f}+(1-\phi) \rho_{s}$ is the mixture density, $\phi$ is the porosity, $\boldsymbol{\sigma}(\boldsymbol{x}, t)$ is the total stress tensor and $p(\boldsymbol{x}, t)$ is the interstitial fluid pressure in the pores; the vectors of displacement of the solid skeleton and of the fluid are denoted by $\boldsymbol{u}_{s}(\boldsymbol{x}, t)$ and $\boldsymbol{u}_{f}(\boldsymbol{x}, t)$, respectively; the vector of relative displacement between the fluid and the solid frame weighted by the porosity is denoted by $\boldsymbol{w}=\phi\left(\boldsymbol{u}_{f}-\boldsymbol{u}_{s}\right) ; \boldsymbol{\kappa}$ is the permeability tensor defined by $\boldsymbol{\kappa}=\boldsymbol{k} / \mu$ where $\boldsymbol{k}$ is the intrinsic permeability tensor and $\mu$ is the fluid's dynamic viscosity; the tensor $\boldsymbol{b}$ is defined as $\boldsymbol{b}=\left(\rho_{f} / \phi\right) \boldsymbol{a}$ where $\boldsymbol{a}$ is the tortuosity tensor. Note that both tensors $\boldsymbol{k}$ and $\boldsymbol{b}$ are symmetric second-order tensors.
The constitutive equations for the anisotropic linear poroelastic material are given by:

$\begin{aligned} \boldsymbol{\sigma} & =\mathbb{C}: \boldsymbol{\epsilon}-\boldsymbol{\alpha} p, \\ -\frac{1}{M} p & =\nabla \cdot \boldsymbol{w}+\boldsymbol{\alpha}: \boldsymbol{\epsilon},\end{aligned}$

where $\mathbb{C}$ is the elasticity fourth-order tensor of drained porous material; $\boldsymbol{\alpha}$, which is a symmetric second-order tensor, is the Biot effective tensor; $M$ is the Biot scalar modulus; $\boldsymbol{\epsilon}(\boldsymbol{x}, t)$ is the infinitesimal strain tensor which is defined as the symmetric part of $\nabla \boldsymbol{u}_{s}$.

\subsubsection{Boundary and interface conditions}

The surface of the soft tissue consists of 2 parts: $\Gamma_{0}=$ $\Gamma_{0}^{P} \cup \Gamma_{0}^{F}$ which correspond to the zone excited by pulsed pressure and the free surface, respectively:

$p_{0}^{f}(\boldsymbol{x}, t)=P_{0} g(t), \quad \forall \boldsymbol{x} \in \Gamma_{0}^{P}$,

$p_{0}^{f}(\boldsymbol{x}, t)=0, \quad \forall \boldsymbol{x} \in \Gamma_{0}^{F}$,

where $P_{0}$ is the amplitude and $g(t)$ is pulsed time function with a central frequency $f_{c}: g(t)=\sin \left(2 \pi f_{c} t\right)$. At interfaces $\Gamma_{j}((j=0, \ldots, N))$, the continuity of pressure and stress fields between the poroealstic medium and the fluid domains imposes:

$\left.\begin{array}{l}p_{f}=p_{j}^{f}, \\ \boldsymbol{\sigma} \boldsymbol{n}_{j}=-p_{j}^{f} \boldsymbol{n}_{j},\end{array}\right\} \quad \forall \boldsymbol{x} \in \Gamma_{j}(j=0, \ldots, N)$,

where $\boldsymbol{n}_{j}$ is the normal unit vector to $\Gamma_{j}$ pointing from the porous domain $\Omega^{b}$ towards the fluid domain $\Omega_{j}^{f}$ (see Fig. 1).

The periosteal interface $\left(\Gamma_{0}\right)$ is assumed to be impervious (Goulet et al., 2008), requiring:

$\boldsymbol{w}=\mathbf{0}, \quad \forall \boldsymbol{x} \in \Gamma_{0}$.

At the other interfaces $\Gamma_{j}(j=1, \ldots, N)$, open-pore condition is assumed, requiring:

$\dot{\boldsymbol{w}} \cdot \boldsymbol{n}_{j}=\left(\boldsymbol{v}_{j}-\dot{\boldsymbol{u}}_{s}\right) \cdot \boldsymbol{n}_{j}, \quad \forall \boldsymbol{x} \in \Gamma_{j}(j=1, \ldots, N)$

In view of the Euler equation (2), the interface condition (11) may be rewritten as:

$\left(\frac{1}{\rho_{j}^{f}} \nabla p_{j}^{f}+\ddot{\boldsymbol{w}}+\ddot{\boldsymbol{u}}_{s}\right) \cdot \boldsymbol{n}_{j}=0, \quad \forall \boldsymbol{x} \in \Gamma_{j}(j=1, \ldots, N)$ 


\subsection{Weak formulation}

\subsubsection{Weak formulation in the fluid domains $\Omega_{j}^{f}$}

The pressure field in the fluid occupying the domain $\Omega_{j}^{f}$ is described by Eq. (1). The weak form of this equation may be obtained by multiplying it by a scalar valued test function $\tilde{p}_{j}^{f}$ (with support in $\Omega_{j}^{f}$ ) and then integrating over the domain $\Omega_{j}^{f}$. By applying the Green-Gauss theorem and taking into account the boundary conditions (12), the weak formulation of the acoustic wave problem in $\Omega_{j}^{f}$ reads:

$$
\begin{aligned}
\int_{\Omega_{j}^{f}} \frac{1}{K_{j}^{f}} \tilde{p}_{j}^{f} \ddot{p}_{j}^{f} & +\int_{\Omega_{j}^{f}} \frac{1}{\rho^{f}} \nabla \tilde{p}_{j}^{f} \cdot \nabla p_{j}^{f} \\
& +\int_{\Gamma_{j}} \tilde{p}_{j}^{f}\left(\ddot{\boldsymbol{w}}+\ddot{\boldsymbol{u}}_{s}\right) \cdot \boldsymbol{n}_{j}=0, \forall \tilde{p}_{j}^{f} \in \mathfrak{C}^{\mathrm{ad}},
\end{aligned}
$$

where $\mathfrak{C}^{\text {ad }}$ is the admissible function space of the problem constituted by the sufficiently differentiable realvalued functions.

Note that the $\boldsymbol{n}_{j}$ is pointing toward the interior of the fluid domain $\Omega_{j}^{f}$.

\subsubsection{Weak formulation in the bone domain $\Omega^{b}$}

In order to resolve the time-dependent problem which is a high frequency problem, the finite element analysis were based on the $\left(\boldsymbol{u}_{s}-\boldsymbol{w}\right)$ formulation. The balance equations of linear momentum (3)-(4) are already written in terms of $\boldsymbol{u}_{s}$ and $\boldsymbol{w}$. Accordingly, constitutive equations (5)-(6) may be restated as:

$$
\boldsymbol{\sigma}=\mathbb{C}_{u} \boldsymbol{\epsilon}+M \boldsymbol{\alpha} \nabla \cdot \boldsymbol{w}
$$

$p=-M(\boldsymbol{\alpha}: \boldsymbol{\epsilon}+\nabla \cdot \boldsymbol{w})$,

where $\mathbb{C}_{u}=\mathbb{C}+M \boldsymbol{\alpha} \otimes \boldsymbol{\alpha}$ is the undrained elasticity tensor. (The symbol $\otimes$ designates the tensorial product between two tensors.)

Let $\tilde{\boldsymbol{u}}_{s}$ and $\tilde{\boldsymbol{w}}$ be two vector valued test functions with support in $\Omega^{b}$. The weak form of balance equations (3)-(4) may be obtained by multiplying them by $\tilde{\boldsymbol{u}}_{s}$ and $\tilde{\boldsymbol{w}}$, respectively, integrating over $\Omega^{b}$ and applying the
Green-Gauss theorem (Nguyen et al., 2010b):

$$
\begin{array}{r}
\int_{\Omega^{b}} \tilde{\boldsymbol{u}}_{s} \cdot \rho \ddot{\boldsymbol{u}}_{s}+\int_{\Omega^{b}} \tilde{\boldsymbol{u}}_{s} \cdot \rho_{f} \ddot{\boldsymbol{w}}+\int_{\Omega^{b}} \nabla \tilde{\boldsymbol{u}}_{s}: \boldsymbol{\sigma} \\
+\sum_{j} \int_{\Gamma_{j}} \tilde{\boldsymbol{u}}_{s} \cdot\left(p_{j}^{f} \boldsymbol{n}_{j}\right)=0, \\
\quad \forall \tilde{\boldsymbol{u}}_{s} \in \mathfrak{C}^{\mathrm{ad}}, \\
\int_{\Omega^{b}} \tilde{\boldsymbol{w}} \cdot \rho_{f} \ddot{\boldsymbol{u}}_{s}+\int_{\Omega^{b}} \tilde{\boldsymbol{w}} \cdot(\boldsymbol{b} \ddot{\boldsymbol{w}})+\int_{\Omega^{b}} \tilde{\boldsymbol{w}} \cdot\left(\boldsymbol{\kappa}^{-1} \dot{\boldsymbol{w}}\right) \\
+\int_{\Omega^{b}} \nabla \tilde{\boldsymbol{w}}:(p \boldsymbol{I})+\sum_{j} \int_{\Gamma_{j}} \tilde{\boldsymbol{w}} \cdot\left(p_{j}^{f} \boldsymbol{n}_{j}\right)=0, \\
\forall \tilde{\boldsymbol{w}} \in \mathfrak{C}^{\mathrm{ad}},
\end{array}
$$

where $\boldsymbol{I}$ is the second-order identity tensor.

\subsection{Computational model}

Ultrasound signal. The ultrasound signal emitted from $\Gamma_{0}^{P}$ (see Fig. 1) is a pulsed signal with a central frequency $f_{c}=1 \mathrm{MHz}$, a duty cycle of $20 \%$ and a repetition frequency $f_{r}=1 \mathrm{kHz}$. The spatial average-time average acoustic intensity delivered, $\mathrm{I}_{\mathrm{SATA}}$, is of $30 \mathrm{~mW} / \mathrm{cm}^{2}$ corresponding to an acoustic pressure amplitude $P_{0}=67 \mathrm{kPa}$ (assuming pressure amplitude is constant over the transducer face). The transducer diameter is equal to $2 \mathrm{~cm}$.

\section{Material properties.}

- Fluid phase: soft tissue, marrow and fluid inside the vascular pores are supposed to be perfect fluids mechanically equivalent to water: speed of sound $c_{f}=1500 \mathrm{~m} / \mathrm{s}$; mass density $\rho_{f}=1000 \mathrm{~kg} / \mathrm{m}^{3} ;$ bulk modulus $K=2.25 \mathrm{GPa}$.

- Poroelastic phase: bone matrix around the vascular pores is considered as a transverse isotropic poroelastic medium. The LCN porosity is taken from the literature $\phi=0.05$ (Smit et al., 2002; Lemaire et al., 2012), the fluid inside the pores is considered as water with a dynamic viscosity $\mu=10^{-3} \mathrm{~Pa}^{-\mathrm{s}^{-1}}$.

Around the LCN, the extra-cellular matrix (ECM) has a mass density $\rho_{\mathrm{ECM}}$ equal to $2 \mathrm{~g} / \mathrm{cm}^{3}$. The model is constructed in two dimensional space in a plane perpendicular to the bone axis where the elastic properties of the ECM are assumed to be isotropic. The elasticity tensor $\mathbb{C}$ in this plane is expressed from the Voigt notation as (Scheiner et al., 2016):

$$
\mathbb{C}=\left(\begin{array}{ccc}
C_{1111} & C_{1122} & 0.0 \\
C_{1122} & C_{1111} & 0.0 \\
0.0 & 0.0 & C_{1212}
\end{array}\right),
$$


with $C_{1212}=\frac{1}{2}\left(C_{1111}-C_{1122}\right)$. The values used in the simulations are $C_{1111}=22.88 \mathrm{GPa}$ and $C_{1122}=$ $10.14 \mathrm{GPa}$ which lead to the Young modulus $E=$ 16.56 GPa and Poisson ratio $\nu=0.308$. The Biot's parameters are calculated from this elasticity tensor. The component values of the Biot effective tensor and the Biot scalar modulus are given by $\alpha_{11}=$ $\alpha_{22}=0.15$ and $M=35.6 \mathrm{GPa}$ (for more details see Rosi et al. (2016)).

Concerning the permeability of this porous medium, two values are investigated. Inspired from the permeability estimation of the vascular porous network in cortical bone (Zhang et al., 1998; Swan et al., 2003), a first value of LCN permeability is calculated from the Kozeny-Carman relation, assuming a Poiseuille flow in a network of cylindrical capillaries aligned in the direction $e_{i}$ of radius $R_{i}=$ $50 \times 10^{-9} \mathrm{~m}$ and representing a porosity $\phi=5 \%$ : $k_{\mathrm{KC}}=\phi R_{i}^{2} / 8=1.56 \times 10^{-17} \mathrm{~m}^{2}$. A second one is taken from literature (Smit et al., 2002), estimation based on the best fit between finite element predictions and experimental data on canine bone: $k_{\mathrm{S}}=2.2 \times 10^{-22} \mathrm{~m}^{2}$.

Computation parameters. The convergence of the numerical results was tested to choose a mesh size $(\Delta x)$ smaller than the tenth of the wavelength $\lambda$ in each material. To ensure a relevant sampling of the timedependent phenomena, the solver time stepping $\Delta t$ was fixed to $\mathrm{T} / 40$ where $\mathrm{T}=1 / f_{c}\left(f_{c}\right.$ is the central frequency of pulsed time function and $\left.\Delta t=2.5 \times 10^{-8} \mathrm{~s}\right)$. Note that this time step value verifies the calculation stability condition $\Delta t<\frac{\Delta x}{\sqrt{2} V_{\max }}$, where $V_{\max }$ is a maximum velocity estimated.

Numerical simulations were run with the commercial finite-element software Comsol Multiphysics.

\section{Results and Discussion}

As it has been widely reported in litterature, the osteocytes are the cornerstone of bone remodelling. One of their main characteristics is that they are mechanosensitive cells (see for instance Bonewald (2011)). It has been demonstrated that the dendritic processes of the osteocytes are more sensitive to mechanical loading than the cell body (Han et al., 2004; Burra et al., 2010) and in particular to fluid shear stress (Bakker et al., 2001; Anderson et al., 2005). Inspired from these assumptions, this study focuses on the IF wall shear stress $\left(\mathrm{WSS}_{\mathrm{IF}}\right)$ induced by the ultrasound stimulation into the canaliculi. However, the present poroelastic approach does not allow to directly assess $\mathrm{WSS}_{\mathrm{IF}}$ acting on individual osteocytes, but we can still calculate the $\mathrm{WSS}_{\mathrm{IF}}$ from the velocity of the interstitial fluid relative to the ECM obtained from the Biot's theory $(\dot{\boldsymbol{w}})$. We do this using two different models of fluid flow associated with two different permeability values of the LCN.

$K C$-model. In line with the first permeability value studied $\left(k_{\mathrm{KC}}\right)$, the wall shear stress $\tau_{\mathrm{KC}}$ can be firstly assessed using Kozeny-Carman theory, through equation (19):

$\tau_{\mathrm{KC}}=\frac{R_{i} \mu|\dot{\boldsymbol{w}}|}{2 k_{\mathrm{KC}}}$,

with $R_{i}$ is the typical radius of canaliculi in the LCN $\left(50 \times 10^{-9} \mathrm{~m}\right), \mu$ is the IF dynamic viscosity $\left(10^{-3}\right.$ Pa.s $\left.{ }^{-1}\right)$, $k_{\mathrm{KC}}$ is the LCN permeability $\left(1.56 \times 10^{-17} \mathrm{~m}^{2}\right)$ and $\dot{\boldsymbol{w}}$ is the velocity of the interstitial fluid relative to the ECM calculated from the Biot's theory (see §2.3.2). Equation (19) assumes that the LCN is modelled as parallel straight tubes full of fluid embedded in a solid matrix.

WT-model. Wall shear stress levels can also be defined from Wang and Tarbell (1995) as:

$\tau_{\mathrm{WT}}=\frac{\mu|\dot{\boldsymbol{w}}|}{\sqrt{k_{\mathrm{S}}}}$

where $k_{\mathrm{S}}$ is the LCN permeability equals to $2.2 \times 10^{-22} \mathrm{~m}^{2}$ (Smit et al., 2002). This equation given in Goulet et al. (2008) considers the fluid flow in an annular space through a net of transverse fibers taking into consideration the presence of the osteocyte process inside the canaliculus and the glycosaminoglycans (GAG) fibers which are transverse fibrils which anchor and center the cell process in its canaliculus (see Fig. 2).

\subsection{KC-model vs WT-model}

We calculate the moving average of the $\mathrm{WSS}_{\mathrm{IF}}$ over a signal period $(1 \mu \mathrm{s})$. Data are smoothed with the "loess" method (LOcally Estimated Scatterplot Smoothing), which is a method using linear regression of smooth data. The method is altered to assign a zero weight to data outside six mean absolute standard deviations. We compare the time evolution of $\mathrm{WSS}_{\mathrm{IF}}\left(\tau_{\mathrm{KC}}\right.$ or $\left.\tau_{\mathrm{WT}}\right)$ over 3 cycles for both LCN permeability values $k_{\mathrm{KC}}$ and $k_{\mathrm{S}}$ (see Fig. 3). In what follows, the quantities $\tau_{\mathrm{KC}}$ and $\tau_{\mathrm{WT}}$ are evaluated in points shown on the bone geometry.

Firstly, the computational model developed in this study provides results on $\mathrm{WSS}_{\mathrm{IF}}$ induced by ultrasound stimulation at cell scale which can be considered as reasonable. Comparing both models (KC and WT) used to 
Poroelasticity model: LCN+ECM = equivalent medium Output parameter: $\dot{w}$

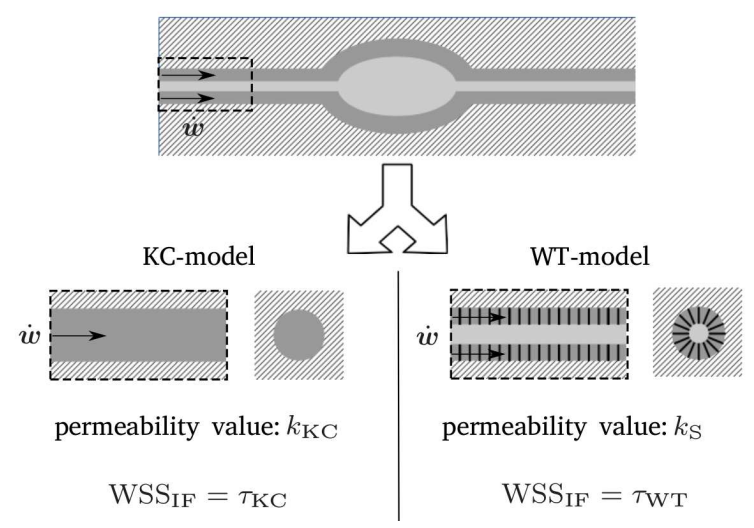

Fig. 2: Schematic methodology to calculate WSS $S_{I F}$ from the output parameter $\dot{\boldsymbol{w}}$ given by the poroelastic theory implemented in the numerical model. Two assumptions are investigated: the KC-model of the fluid flow inside the canaliculus, ignoring the existence of the osteocyte process,

surrounded by the ECM following the Kozeny-Carman relation (left) and the WT-model including the process and the GAG fibers immersed in interstitial fluid to match up to relation given by Wang and Tarbell (1995) (right). The osteocytes is in light gray, the interstitial fluid in dark gray and the GAG fibers in black; the ECM is hatched.

represent the canalicular flow, a very significant difference can be observed: the $\mathrm{WSS}_{\mathrm{IF}}$ levels $\tau_{\mathrm{KC}}$, range from 0 to $600 \mathrm{~Pa}$ i.e. 3 orders of magnitude higher than those of $\tau_{\mathrm{WT}}$ between 0 and $0.6 \mathrm{~Pa}$. It is therefore clear that the way to calculate $\mathrm{WSS}_{\mathrm{IF}}$ in the porous medium has a great influence on the results. In comparison with literature, only the WT-model provides $\mathrm{WSS}_{\mathrm{IF}}$ values in agreement with a potential mechanical signal to activate osteocyte: $0.8<\tau<3 \mathrm{~Pa}$ (Weinbaum et al., 1994), even if they are close to the lower limit of the interval. These results confirm that the KC-model, bundle of aligned cylindrical pores full of fluid, neglecting the presence of the process, is inappropriate to correctly represent the interstitial fluid behavior in the LCN, as it has already been proven by Weinbaum et al. (1994). They showed that one has to consider the fibers surrounding the cell process. The WT-model, firstly developed to model the flow through muscle cells, has been already used for modeling the fluid shear stress inside the LCN of the cortical bone (Goulet et al., 2008) using the analogy of the system osteocyte process and GAG fibers with muscle cell and muscle fibers but also in scaffolds designed for bone regeneration (Ouyang et al., 2019; Ali and Sen, 2018) including under LIPUS (Wu et al., 2015).

According to these observations, only results from the WT-model are analysed further.

\subsection{Focus on WT-model}

$W S S_{I F}$ distribution over 10 cycles. The stimulation duration is extended to 10 cycles $(10 \mathrm{~ms})$ for the WTmodel. On Fig. 4, the $\mathrm{WSS}_{\mathrm{IF}}$ distribution is represented for three different times: $1 \mathrm{~ms}, 5 \mathrm{~ms}$ and $10 \mathrm{~ms}$. The most stimulated areas are located around the endosteum and the vascular pores interfaces. Globally the WSS $_{\text {IF }}$ levels increase with time. To further explore evolution in time of the WSS $_{\text {IF }}$ potentially experienced by the osteocytes, $\tau_{\mathrm{WT}}$ is evaluated on 12 points distributed on the bone surface during 10 cycles (see Fig. 5). The curves are obtained in the same procedure as described in $\S 3.2$. Some points, located near the endosteum interface and nearby the vascular pores, reach higher $\tau_{\mathrm{WT}}$ values than the six points investigated in Fig. 3: $0<\tau_{\mathrm{WT}}<1.5 \mathrm{~Pa}$, still in the range defined by Weinbaum et al. (1994). This $\mathrm{WSS}_{\mathrm{IF}}$ distribution may raise questions about the possible role of lining cells into the bone remodelling stimulated by ultrasound. Lining cells are bone cells covering over $90 \%$ of the surfaces of adult bone, and which are also recognized as mecanosensory cells (Mullender and Huiskes, 1997; Robling and Turner, 2009). They are liable to play a role in bone remodelling triggering, especially since ultrasonic stimulation transmits compression waves perpendicular to the bone axis and thus a form of mechanical stress different from physiological loading, preferentially oriented parallel to the bone axis.

The obtained values (see Fig. 5) and the spatial heterogeneity of the WSS $_{\text {IF }}$ distribution (see Fig. 4) are in agreement with the results reported in Fan et al. (2016) on mouse bone with a similar canalicular flow model as the WT-model, even if the loading conditions are not the same, in particular, the frequency loading (around $0.5 \mathrm{~Hz}$ ) which is lower than the pulsed ultrasound frequencies.

Another factor that can influence the distribution of the mechanical stimulus is absorption that could lead to wave attenuation. The Biot's poroelastic model used in this work to represent the interaction of the LCN with ultrasonic waves assumes that the solid phase is elastic and the fluid is viscous $\left(\mu=10^{-3} \mathrm{~Pa} / \mathrm{s}\right)$. This absorption is taken into account via the fluid's dynamic viscosity in the permeability tensor $(\alpha)$. On the other hand, absorption related to the solid phase of the porous medium is neglected in this first approach.

Frequency loading dependency. Indeed, the frequency of cyclic loading is regarded as a decisive factor for bone formation (Weinbaum et al., 1994; Bacabac et al., 2004; Klein-Nulend et al., 2005; González-Torres et al., 2010; Gómez-Benito et al., 2011). It is noteworthy that in 

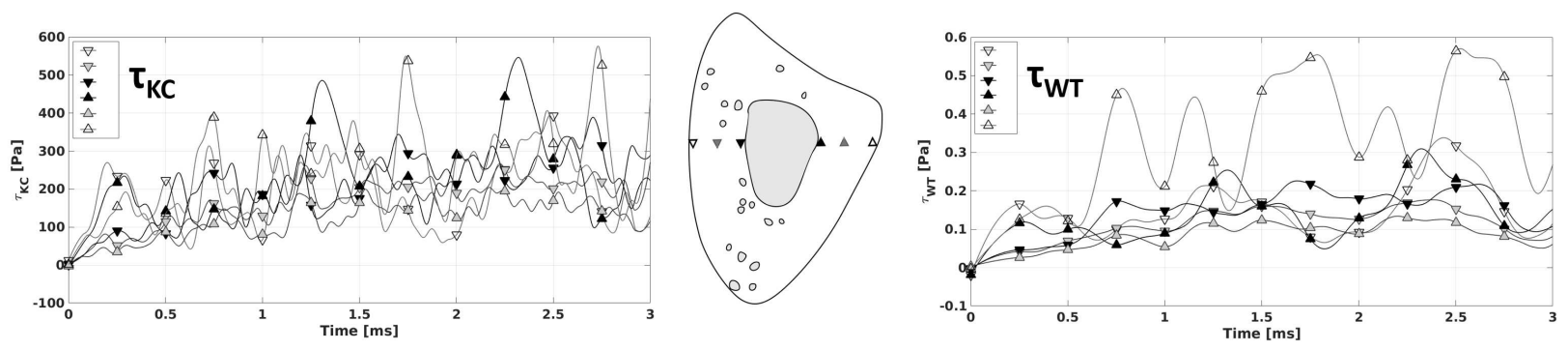

Fig. 3: WSS IF estimated in 6 points over 3 cycles (3 ms) for (a) KC-model and (b) WT-model.

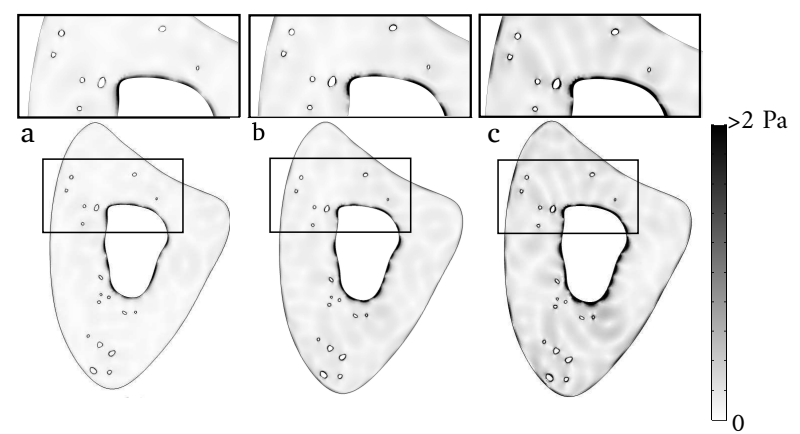

Fig. 4: WSS $S_{I F}$ estimated for WT-model at a) $1 \mathrm{~ms}$, b) $5 \mathrm{~ms}$ and c) $10 \mathrm{~ms}$ (same color scale).
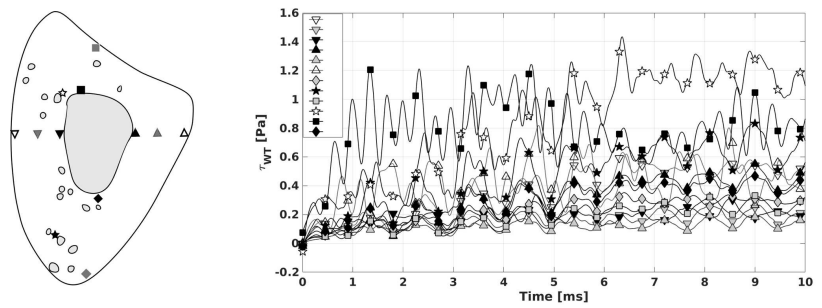

Fig. 5: WSS IF estimated for WT-model in 12 points over 10 cycles $(10 \mathrm{~ms})$.

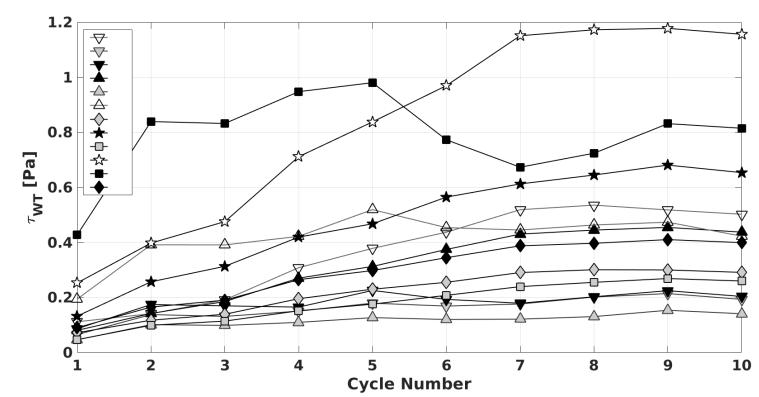

Fig. 6: $W S S_{I F}$ averaged over each of the ten first cycles.

our case, two frequencies can be considered and can play a role in the bone cell stimulation: the frequency of the ultrasonic signal, $1 \mathrm{MHz}$; and the pulse repeti- tion frequency, $1 \mathrm{kHz}$. Both are much higher than the physiological loading frequencies $(1-20 \mathrm{~Hz})$. However, it has been demonstrated that a same load applied on bone at mesoscopic scale, at high frequency, could induce sufficient stress on the osteocytes to activate a biological response when it would have had no effect at low frequencies (Weinbaum et al., 1994; Han et al., 2004). However the frequency range $(0-100 \mathrm{~Hz})$ of these observations remains below the two frequencies considered here. Following Bacabac et al. (2004), one of the determinant parameter for osteocytes response is the rate of fluid shear stress (the product of fluid shear stress amplitude in $\mathrm{Pa}$ and frequency loading in $\mathrm{Hz}$ ). In their work on cell culture in a flow chamber, they assumed that low-magnitude, high-frequency mechanical stimuli may be as stimulatory as high-amplitude, low-frequency stimuli. In the present work, assuming that the trend remains similar for frequencies beyond $9 \mathrm{~Hz}$ (maximum frequency studied in Bacabac et al. (2004)), it would mean that considering the signal frequency of $1 \mathrm{MHz}$, a fluid shear stress magnitude of $50 \mu \mathrm{Pa}$ could be sufficient to activate an osteogenic response, and considering the pulse repetition frequency of $1 \mathrm{kHz}$, the fluid shear stress magnitude reliable to induce osteocyte response should be around $0.05 \mathrm{~Pa}$. In both cases, $\tau_{\mathrm{WT}}$ is higher than these levels of fluid shear stress.

Stimulation duration. One of the limitations of this study is that the maximum stimulation duration considered is $10 \mathrm{~ms}$ which is much shorter than the daily treatment time $(20 \mathrm{~min})$ defined in the fracture repair protocols (Poolman et al., 2017). Consequently, one of the points to check is that there are no cumulative effects during stimulation cycles. On Fig. 5, it can be seen that values obtained on the chosen points are quite stable over time. This trend is confirmed on Fig. 6 which shows the value of $\tau_{W T}$ averaged per cycle of $1 \mathrm{~ms}$ for each considered point. Nevertheless, further works are needed to resolve technical issues (simulation time and file size) and to look at longer-time phenomena. 


\section{Conclusion}

This work provides a numerical model of the interaction of low pulsed intensity ultrasound and cortical bone as a two-level porous medium. The main objective of this study is to gain insight into the process of mechanotransduction induced by ultrasound stimulation. Considering the osteocytes as the pilot of the bone remodelling under mechanical stimuli, the modelling of the LCN was essential. The reconstruction of this porosity network from images not being available over the entire surface considered, the model uses the theory of poroelasticity to assess the physical quantities relative to this porosity scale and estimate the effect of ultrasonic waves at the cell scale. The results obtained tend to prove the potential of the model detailed in this study to represent the interaction of ultrasound stimulation and cortical bone taking into account two levels of porosity. Furthermore, they highlight how the value of the LCN permeability could affect the $\mathrm{WSS}_{\mathrm{IF}}$ levels. According to literature, it tends to demonstrate that the $\mathrm{KC}$-model is not relevant to represent the fluid flow into the LCN and that the WT-model seems to be more appropriate to evaluate the $\mathrm{WSS}_{\mathrm{IF}}$ levels potentially experienced by the osteocytes.

Nevertheless, most of the assumptions have been interpolated from previous works on bone mechanotransduction under physiological loading, more data are needed on osteocyte activation under higher frequency stimulation to identify the physical and biological processes at work in the context of pulsed ultrasound stimulation. Further studies are on going to investigate the influence of US parameters on the mechanical signal received by osteocytes and explore the effect of the multiscale bone anisotropy through a 3D-model including the absorption of the solid phase of the bone matrix. Investigations are under progress to experimentally validate the model.

\section{References}

D. Ali and S. Sen. Permeability and fluid flow-induced wall shear stress of bone tissue scaffolds: computational fluid dynamic analysis using newtonian and non-newtonian blood flow models. Computers in Biology and Medicine, 99:201-208, 2018.

E. J. Anderson, S. Kaliyamoorthy, J. Iwan, D. Alexander, and M. L. Knothe Tate. Nano-microscale models of periosteocytic flow show differences in stresses imparted to cell body and processes. Annals of Biomedical Engineering, 33(1):52-62, 2005.
P. Aspenberg. Comment to a BMJ Editorialls LIPUS the baby in the bathwater? Acta Orthopaedica, 88 (1):1, 2017.

R. G. Bacabac, T. H. Smit, M. G. Mullender, S. J. Dijcks, J. J. W. A. Van Loon, and J. Klein-Nulend. Nitric oxide production by bone cells is fluid shear stress rate dependent. Biochemical and Biophysical Research Communications, 315(4):823-829, 2004.

A. D. Bakker, K. Soejima, J. Klein-Nulend, and E. H. Burger. The production of nitric oxide and prostaglandin E2 by primary bone cells is shear stress dependent. Journal of Biomechanics, 34(5):671-677, 2001.

M. A. Biot. Theory of elasticity and consolidation for a porous anisotropic solid. Journal of Applied Physics, 26(2):182-185, 1955.

M. A. Biot. The elastic coefficients of the theory of consolidation. Journal of Applied Mechanics, 24:594601, 1957.

L. F. Bonewald. The amazing osteocyte. Journal of Bone and Mineral Research, 26(2):229-238, 2011.

S. Burra, D. P. Nicolella, W. L. Francis, C. J. Freitas, N. J. Mueschke, K. Poole, and J. X. Jiang. Dendritic processes of osteocytes are mechanotransducers that induce the opening of hemichannels. Proceedings of the National Academy of Sciences of the United States of America, 107(31):13648-13653, 2010.

J. W. Busse, M. Bhandari, T. A. Einhorn, J. D. Heckman, K-S. Leung, E. Schemitsch, P. Tornetta, S. D. Walter, and G. H. Guyatt. Trial to re-evaluate ultrasound in the treatment of tibial fractures (TRUST): a multicenter randomized pilot study. Trials, 15:206, 2014.

J. A. Buza and T. Einhorn. Bone healing in 2016. Clinical Cases in Mineral and Bone Metabolism, 13:101105, 2016.

L. Cardoso, S. P. Fritton, G. Gailani, M. Benalla, and S. C. Cowin. Advances in assessment of bone porosity, permeability and interstitial fluid flow. Journal of Biomechanics, 46(2):253-265, 2013.

C. W. Chan, L. Qin, K. M. Lee, M. Zhang, J. C. Y. Cheng, and Kwok S. Leung. Low intensity pulsed ultrasound accelerated bone remodeling during consolidation stage of distraction osteogenesis. Journal of Orthopaedic Research: Official Publication of the Orthopaedic Research Society, 24(2):263-270, 2006.

L. E. Claes and C. A. Heigele. Magnitudes of local stress and strain along bony surfaces predict the course and type of fracture healing. Journal of Biomechanics, 32 (3):255-266, 1999.

L. E. Claes and B. Willie. The enhancement of bone regeneration by ultrasound. Progress in Biophysics and Molecular Biology, 93(1-3):384-398, 2007. 
C. Corradi and A. Cozzolino. Effect of ultrasonics on the development of osseous callus in fractures. Archivio Di Ortopedia, 66(1):77-98, 1953.

O. Coussy. Thermoporoelasticity. In Poromechanics, pages 71-112. John Wiley \& Sons, Ltd, 2005.

S. C. Cowin. A recasting of anisotropic poroelasticity in matrices of tensor components. Transport in Porous Media, 50(1):35-56, 2003.

S. C. Cowin, L. Moss-Salentijn, and M. L. Moss. Candidates for the mechanosensory system in bone. Journal of Biomechanical Engineering, 113(2):191-197, 1991.

L. R. Duarte. The stimulation of bone growth by ultrasound. Archives of orthopaedic and traumatic surgery, 101(3):153-159, 1983.

L. Fan, S. Pei, X. Lucas L., and L. Wang. A multiscale 3d finite element analysis of fluid/solute transport in mechanically loaded bone. Bone Research, 4:16032, 2016.

L. A. González-Torres, M. J. Gómez-Benito, M. Doblaré, and J. M. García-Aznar. Influence of the frequency of the external mechanical stimulus on bone healing: a computational study. Medical Engineering \& Physics, 32(4):363-371, 2010.

G. C. Goulet, N. Hamilton, D. Cooper, D. Coombe, D. Tran, R. Martinuzzi, and R. F. Zernicke. Influence of vascular porosity on fluid flow and nutrient transport in loaded cortical bone. Journal of Biomechanics, 41(10):2169-2175, 2008.

X. L. Griffin. Low intensity pulsed ultrasound for fractures of the tibial shaft. BMJ, 355:i5652, 2016.

M. J. Gómez-Benito, L. A. González-Torres, E. ReinaRomo, J. Grasa, B. Seral, and J. M. García-Aznar. Influence of high-frequency cyclical stimulation on the bone fracture-healing process: mathematical and experimental models. Philosophical Transactions of the Royal Society of London A: Mathematical, Physical and Engineering Sciences, 369(1954):4278-4294, 2011.

Y. Han, S. C. Cowin, M. B. Schaffler, and S. Weinbaum. Mechanotransduction and strain amplification in osteocyte cell processes. Proceedings of the National Academy of Sciences, 101(47):16689-16694, 2004.

H. Isaksson, W. Wilson, C. C. van Donkelaar, R. Huiskes, and K. Ito. Comparison of biophysical stimuli for mechano-regulation of tissue differentiation during fracture healing. Journal of Biomechanics, 39(8):1507-1516, 2006.

J. Klein-Nulend, A. van der Plas, C. M. Semeins, N. E. Ajubi, J. A. Frangos, P. J. Nijweide, and E. H. Burger. Sensitivity of osteocytes to biomechanical stress in vitro. The FASEB Journal, 9(5):441-445, 1995.
J. Klein-Nulend, R. G. Bacabac, and M. G. Mullender. Mechanobiology of bone tissue. Pathologie-Biologie, 53(10):576-580, 2005.

J. Klein-Nulend, A. D. Bakker, R. G. Bacabac, A. Vatsa, and S. Weinbaum. Mechanosensation and transduction in osteocytes. Bone, 54(2):182-190, 2013.

D. Lacroix and P. J. Prendergast. A mechanoregulation model for tissue differentiation during fracture healing: analysis of gap size and loading. Journal of Biomechanics, 35(9):1163-1171, 2002.

T. Lemaire, S. Lemonnier, and S. Naili. On the paradoxical determinations of the lacuno-canalicular permeability of bone. Biomech. Model. Mechanobiol., 11:933-946, 2012.

P. Martinez de Albornoz, A. Khanna, U. G. Longo, F. Forriol, and N. Maffulli. The evidence of lowintensity pulsed ultrasound for in vitro, animal and human fracture healing. British Medical Bulletin, 100 (1):39-57, 2011.

S. M. J. Mortazavi, S. A. R. Mortazavi, and M. Paknahad. Mode \& mechanism of low intensity pulsed ultrasound (LIPUS) in fracture repair. Ultrasonics, 71:142, 2016.

M. G. Mullender and R. Huiskes. Osteocytes and bone lining cells: which are the best candidates for mechano-sensors in cancellous bone? Bone, 20(6): 527-532, 1997.

M. G. Mullender, A. J. El Haj, Y. Yang, M. A. van Duin, E. H. Burger, and J. Klein-Nulend. Mechanotransduction of bone cells in vitro: mechanobiology of bone tissue. Medical \& Biological Engineering 83 Computing, 42(1):14-21, 2004.

V.-H. Nguyen and S. Naili. Simulation of ultrasonic wave propagation in anisotropic poroelastic bone plate using hybrid spectral/finite element method. International Journal for Numerical Methods in Biomedical Engineering, 28(8):861-876, 2012.

V.-H. Nguyen, T. Lemaire, and S. Naili. Numerical study of deformation-induced fluid flows in periodic osteonal matrix under harmonic axial loading. $C . R$. Mecanique, 337:268-276, 2009.

V.-H. Nguyen, T. Lemaire, and S. Naili. Poroelastic behaviour of cortical bone under harmonic axial loading: A finite element study at the osteonal scale. Medical Engineering \& Physics, 32:384-390, 2010a.

V.-H. Nguyen, V. Sansalone, and S. Naili. Simulation of ultrasonic wave propagation in anisotropic cancellous bone immersed in fluid. Wave Motion, 47(2):117-129, 2010b.

V.-H. Nguyen, T. Lemaire, and S. Naili. Influence of interstitial bone microcracks on strain-induced fluid flow. Biomechanics and Modeling in Mechanobiology, 
10:963-972, 2011.

P. Ouyang, H. Dong, X. He, X. Cai, Y. Wang, J. Li, H. Li, and Z. Jin. Hydromechanical mechanism behind the effect of pore size of porous titanium scaffolds on osteoblast response and bone ingrowth. $\mathrm{Ma}$ terials \& Design, 183:108151, 2019.

F. Padilla, R. Puts, L. Vico, and K. Raum. Stimulation of bone repair with ultrasound: a review of the possible mechanic effects. Ultrasonics, 54(5):1125-1145, 2014.

R. Poolman, T. Agoritsas, R. Siemieniuk, I. Harris, I. Schipper, B. Mollon, M. Smith, A. Albin, S. Nador, W. Sasges, S. Schandelmaier, L. Lytvyn, T. Kuijpers, L. Van Beers, M. Verhofstad, and P. Vandvik. Low intensity pulsed ultrasound (LIPUS) for bone healing: A clinical practice guideline. BMJ, 356:j576, 2017.

P. J. Prendergast, R. Huiskes, and K. Søballe. Biophysical stimuli on cells during tissue differentiation at implant interfaces. Journal of Biomechanics, 30 (6):539-548, 1997.

A. G. Robling and C. H. Turner. Mechanical Signaling for Bone Modeling and Remodeling. Critical reviews in eukaryotic gene expression, 19(4):319-338, 2009.

G. Rosi, V.-H. Nguyen, and S. Naili. Numerical investigations of ultrasound wave propagating in long bones using a poroelastic model. Mathematics and Mechanics of Solids, 21(1):119-133, 2016.

S. Schandelmaier, A. Kaushal, L. Lytvyn, D. HeelsAnsdell, R. A. C. Siemieniuk, T. Agoritsas, G. H. Guyatt, P. O. Vandvik, R. Couban, B. Mollon, and J. W. Busse. Low intensity pulsed ultrasound for bone healing: systematic review of randomized controlled trials. BMJ, 356:j656, 2017.

S. Scheiner, P. Pivonka, and C. Hellmich. Poromicromechanics reveals that physiological bone strains induce osteocyte-stimulating lacunar pressure. Biomechanics and Modeling in Mechanobiology, 15(1):9-28, 2016.

J. Schortinghuis, B. Stegenga, G. M. Raghoebar, and L. G. M. de Bont. Ultrasound stimulation of maxillofacial bone healing. Critical Reviews in Oral Biology and Medicine, 14(1):63-74, 2003.

T. H. Smit, J. M. Huyghe, and S. C. Cowin. Estimation of the poroelastic parameters of cortical bone. Journal of Biomechanics, 35(6):829-835, 2002.

C. C. Swan, R. S. Lakes, R. A. Brand, and K. J. Stewart. Micromechanically based poroelastic modeling of fluid flow in haversian bone. Journal of Biomechanical Engineering, 125(1):25-37, 2003.

M. Thompson and J. R. Willis. A reformation of the equations of anisotropic poroelasticity. Journal of Applied Mechanics, 58(3):612-616, 1991.
D. M. Wang and J. M. Tarbell. Modeling interstitial flow in an artery wall allows estimation of wall shear stress on smooth muscle cells. Journal of Biomechanical Engineering, 117(3):358-363, 1995.

S. Weinbaum, S. C. Cowin, and Yu Zeng. A model for the excitation of osteocytes by mechanical loadinginduced bone fluid shear stresses. Journal of Biomechanics, 27(3):339-360, 1994.

L. Wu, L. Lin, and Y.-X. Qin. Enhancement of cell ingrowth, proliferation, and early differentiation in a three-dimensional silicon carbide scaffold using lowintensity pulsed ultrasound. Tissue Engineering. Part A, 21(1-2):53-61, 2015.

I. Yasuda. The classic: Fundamental aspects of fracture treatment by Iwao Yasuda, reprinted from J. Kyoto Med. Soc., 4:395-406, 1953. Clinical Orthopaedics and Related Research, 124:5-8, 1977.

D. Zhang, S. Weinbaum, and S. C. Cowin. Estimates of the peak pressures in bone pore water. Journal of Biomechanical Engineering, 120(6):697-703, 1998. 